\title{
THE SECOND VARIATION FORMULA FOR EXPONENTIALLY HARMONIC MAPS
}

\author{
Leung-Fu Cheung and Pui-Fai Leung
}

\begin{abstract}
We derive the formula in the title and deduce some consequences. For example we show that the identity map from any compact manifold to itself is always stable as an exponentially harmonic map. This is in sharp contrast to the harmonic or p-harmonic cases where many such identity maps are unstable. We also prove that an isometric and totally geodesic immersion of $S^{m}$ into $S^{n}$ is an unstable exponentially harmonic map if $m \neq n$ and is a stable exponentially harmonic map if $m=n$.
\end{abstract}

\section{INTRODUCTION}

A map $f: M^{m} \rightarrow N^{n}$ where $M^{m}$ and $N^{n}$ are compact Riemannian manifolds is called an exponentially harmonic map if it is a critical point of the exponential energy functional.

$$
E(f)=\int_{M} \exp \left(\frac{1}{2}\|d f\|^{2}\right) d v_{M}
$$

In a recent paper [1] Hong has calculated the first variation formula for exponentially harmonic maps and studied some of their properties.

In this note we shall derive in Section 2 the second variation formula for exponentially harmonic maps. We shall use this formula to prove in Section 3 the following theorem which is in sharp contrast to the harmonic and p-harmonic cases.

THEOREM 1. The identity map from any compact Riemannian manifold $M^{m}$ to itself is always stable as an exponentially harmonic map.

Recall that there are many unstable identity maps for the harmonic [4] and pharmonic [5] cases.

In Section 4 we shall study unstable exponentially harmonic maps and shall prove the following result which gives many examples of unstable exponentially harmonic maps.

THEOREM 2. Let $f: S^{m} \rightarrow S^{n}$ be an isometric and totally geodesic immersion from a m-dimensional unit sphere to a n-dimensional unit sphere. Then $f$ is an unstable exponentially harmonic map if $m \neq n$ and is a stable exponentially harmonic map if $m=n$.

Received 4th January, 1999

We would like to thank the referee for many helpful suggestions.

Copyright Clearance Centre, Inc. Serial-fee code: 0004-9729/99 \$A2.00+0.00. 
We are much indebted to the paper [1] which introduced us to the study of exponentially harmonic maps.

\section{The Second Variation Formula}

In this section we shall use the elegant method in [2, pp.20-25] to calculate the second variation of $E(f)$.

Let $g$ denote the Riemannian metric tensor and $\nabla$ denote the Levi-Civita connection on $N^{n}$.

Let $V$ be a vector field on $N^{n}$ and let $\phi_{t}: N^{n} \rightarrow N^{n}$ be the one-parameter group of transformations on $N^{n}$ generated by $V$. Let $f_{t}=\phi_{t} \circ f$ and put

$$
E_{V}(t)=\int_{M} \exp \left(\frac{1}{2}\left\|d f_{t}\right\|^{2}\right) d v_{M}
$$

We wish to calculate $E_{V}^{\prime}(0)$ and $E_{V}^{\prime \prime}(0)$.

Define two tensor fields $A^{V}$, and $\nabla_{V, \bullet} V \in \operatorname{Hom}(T N, T N)$ by

$$
A^{V}(X)=\nabla_{X} V \text { and } \nabla_{V, X} V=\nabla_{V} \nabla_{X} V-\nabla_{\nabla_{V} X} V
$$

We shall now divide our calculations into several steps in the form of a sequence of propositions.

The next two propositions follow from definition.

Proposition 3. $\nabla_{V}-A^{V}=L_{V}$ where $L_{V}$ denotes the Lie derivative with respect to $V$.

Proposition 4. $\quad \nabla_{V, X} V=A^{A^{V}(V)}(X)-A^{V}\left(A^{V}(X)\right)+R(V, X) V$ where $R(V, X) V=\nabla_{V} \nabla_{X} V-\nabla_{X} \nabla_{V} V-\nabla_{[V, X]} V$ is the Riemannian curvature tensor on $N$.

Proposition 5. $\left(L_{V}(g)\right)(X, X)=2 g\left(A^{V}(X), X\right)$.

Proof: By Proposition 3 and the facts that $\nabla g=0$ and $A^{V}=0$ on functions, we have

$$
\left(L_{V}(g)\right)(X, X)=\left(-A^{V}(g)\right)(X, X)=2 g\left(A^{V}(X), X\right)
$$

\footnotetext{
Proposition 6. $\left(L_{V}^{(2)}(g)\right)(X, X)=2 g\left(A^{A^{V}(V)}(X), X\right)+2 g(R(V, X) V, X)+$ $2\left\|A^{V}(X)\right\|^{2}$.
} 
Proof: Using Proposition 3 and Proposition 4 we have

$$
\begin{aligned}
\left(L_{V}^{(2)}(g)\right)(X, X)= & {\left[\left(\nabla_{V}-A^{V}\right)\left(\nabla_{V}-A^{V}\right) g\right](X, X) } \\
= & {\left[-\nabla_{V}\left(A^{V} g\right)+A^{V} A^{V} g\right](X, X) } \\
= & -\nabla_{V}\left[\left(A^{V} g\right)(X, X)\right]+2\left(A^{V} g\right)\left(\nabla_{V} X, X\right) \\
& +A^{V}\left[\left(A^{V} g\right)(X, X)\right]-2\left(A^{V} g\right)\left(A^{V}(X), X\right) \\
= & 2 \nabla_{V}\left(g\left(A^{V}(X), X\right)\right)-2 g\left(A^{V} \nabla_{V} X, X\right)-2 g\left(\nabla_{V} X, A^{V}(X)\right) \\
& -2 A^{V}\left(g\left(A^{V}(X), X\right)\right)+2 g\left(A^{V} A^{V}(X), X\right)+2 g\left(A^{V}(X), A^{V}(X)\right) \\
= & 2 g\left(\nabla_{V} A^{V}(X), X\right)-2 g\left(A^{V} \nabla_{V} X, X\right)+2 g\left(A^{V} A^{V}(X), X\right) \\
& +2 g\left(A^{V}(X), A^{V}(X)\right) \\
= & 2 g\left(\nabla_{V, X} V, X\right)+2 g\left(A^{V} A^{V}(X), X\right)+2 g\left(A^{V}(X), A^{V}(X)\right) \\
= & 2 g\left(A^{A^{V}(V)}(X), X\right)+2 g(R(V, X) V, X)+2\left\|A^{V}(X)\right\|^{2} .
\end{aligned}
$$

Proposition 7. (First Variation Formula)

$$
E_{V}^{\prime}(0)=\int_{M}\left[\exp \left(\frac{1}{2}\|d f\|^{2}\right)\right]\left[\sum_{i=1}^{m} g\left(A^{V}\left(f_{*} e_{i}\right), f_{*} e_{i}\right)\right] d v_{M}
$$

where $\left\{e_{1}, \cdots, e_{m}\right\}$ is a local orthonormal basis on $M^{m}$.

Proof: We have

$$
E_{V}(t)=\int_{M} \exp \left[\sum_{i=1}^{m} \frac{1}{2}\left(\phi_{i}^{*} g\right)\left(f_{*} e_{i}, f_{*} e_{i}\right)\right] d v_{M}
$$

and hence using Proposition 5, we calculate

$$
\begin{aligned}
E_{V}^{\prime}(0) & =\int_{M}\left[\exp \left(\frac{1}{2}\|d f\|^{2}\right)\right]\left[\frac{1}{2} \sum_{i=1}^{m}\left(L_{V}(g)\right)\left(f_{*} e_{i}, f_{*} e_{i}\right)\right] d v_{M} \\
& =\int_{M}\left[\exp \left(\frac{1}{2}\|d f\|^{2}\right)\right]\left[\sum_{i=1}^{m} g\left(A^{V}\left(f_{*} e_{i}\right), f_{*} e_{i}\right)\right] d v_{M}
\end{aligned}
$$

DEFINITION 8: A map $f: M^{m} \rightarrow N^{n}$ is called an exponentially harmonic map if $E_{V}^{\prime}(0)=0$ for all vector fields $V$ on $N^{n}$.

Proposition 9. Let $f: M^{m} \rightarrow N^{n}$ be an isometric immersion. Then the following statements are equivalent :

(a) $f$ is exponentially harmonic. 
(b) $f$ is harmonic.

(c) $f$ is $p$-harmonic for every $p \geqslant 2$.

(d) $f$ is a minimal immersion.

Proof: For an isometric immersion, Proposition 7 gives

$$
E_{V}^{\prime}(0)=\int_{M}\left[\exp \left(\frac{1}{2} m\right)\right]\left[\sum_{i=1}^{m} g\left(A^{V}\left(e_{i}\right), e_{i}\right)\right] d v_{M}
$$

which is a constant $\left(=e^{m / 2}\right)$ multiple of the first variation of the Dirichlet energy functional of $f$ and is also a constant $\left(=\left(e^{m / 2}\right) / m^{(p-2 / 2)}\right)$ multiple of the first variation of the p-energy functional of $f[5, \mathrm{p} .319]$.

Hence we have $(a) \Longleftrightarrow(b) \Longleftrightarrow(c)$.

The fact that $(\mathrm{b}) \Longleftrightarrow(\mathrm{d})$ is well-known.

Proposition 10. (Second Variation Formula) Let $f: M^{m} \rightarrow N^{n}$ be an exponentially harmonic map. Then

$$
\begin{array}{r}
E_{V}^{\prime \prime}(0)=\int_{M}\left[\exp \left(\frac{1}{2}\|d f\|^{2}\right)\right]\left\{\left[\sum_{i=1}^{m} g\left(A^{V}\left(f_{*} e_{i}\right), f_{*} e_{i}\right)\right]^{2}+\sum_{i=1}^{m}\left\|A^{V}\left(f_{*} e_{i}\right)\right\|^{2}\right. \\
\left.+\sum_{i=1}^{m} g\left(R\left(V, f_{*} e_{i}\right) V, f_{*} e_{i}\right)\right\} d v_{M}
\end{array}
$$

Proof: As in the proof of Proposition 7 we have, using Proposition 6,

$$
\begin{aligned}
E_{V}^{\prime \prime}(0)= & \int_{M}\left[\exp \left(\frac{1}{2}\|d f\|^{2}\right)\right]\left\{\left[\sum_{i=1}^{m} g\left(A^{V}\left(f_{*} e_{i}\right), f_{*} e_{i}\right)\right]^{2}\right. \\
+ & \left.+\frac{1}{2} \sum_{i=1}^{m}\left(L_{V}^{(2)}(g)\right)\left(f_{*} e_{i}, f_{*} e_{i}\right)\right\} d v_{M} \\
= & \int_{M}\left[\exp \left(\frac{1}{2}\|d f\|^{2}\right)\right]\left\{\left[\sum_{i=1}^{m} g\left(A^{V}\left(f_{*} e_{i}\right), f_{*} e_{i}\right)\right]^{2}+\sum_{i=1}^{m} g\left(A^{A^{V}(V)}\left(f_{*} e_{i}\right), f_{*} e_{i}\right)\right. \\
& \left.+\sum_{i=1}^{m}\left\|A^{V}\left(f_{*} e_{i}\right)\right\|^{2}+\sum_{i=1}^{m} g\left(R\left(V, f_{*} e_{i}\right) V, f_{*} e_{i}\right)\right\} d v_{M}
\end{aligned}
$$

By Proposition 7 we have, since $f$ is exponentially harmonic,

$$
\int_{M}\left[\exp \left(\frac{1}{2}\|d f\|^{2}\right)\right]\left[\sum_{i=1}^{m} g\left(A^{A^{v}(V)}\left(f_{*} e_{i}\right), f_{*} e_{i}\right)\right] d v_{M}=E_{A^{v}(v)}^{\prime}(0)=0 .
$$

DEFINITION 11: An exponentially harmonic map $f: M^{m} \rightarrow N^{n}$ is said to be stable if $E_{V}^{\prime \prime}(0) \geqslant 0$ for all vector fields $V$ on $N^{n}$. 


\section{EXAMPLES OF STABLE EXPONENTIALLY HARMONIC MAPS}

Our first example is a direct consequence of Proposition 10.

EXAMPLE 12. If $N^{n}$ has non-positive sectional curvature, then every exponentially harmonic map $f: M^{m} \rightarrow N^{n}$ is stable.

TheOREM 13. Let $f: M^{m} \rightarrow M^{m}$ be an isometry. Then $E_{V}^{\prime \prime}(0) \geqslant 0$ for all vector fields $V$ on $M^{m}$ and $E_{V}^{\prime \prime}(0)=0$ if and only if $V$ is a Killing vector field. Hence $f$ is a stable exponentially harmonic map. In particular the identity map from any compact Riemannian manifold to itself is always a stable exponentially harmonic map.

PROOF: Since $f$ is totally geodesic it is an exponentially harmonic map by Proposition 9. Now we look at the second variation formula. As $f$ is an isometry, $\left\{f_{*} e_{1}, \cdots f_{*} e_{m}\right\}$ also forms an orthonormal basis for $M^{m}$.

Therefore we have

$$
E_{V}^{\prime \prime}(0)=e^{m / 2} \int_{M}\left\{(\operatorname{div}(V))^{2}+\|\nabla V\|^{2}-\operatorname{Ric}(V, V)\right\} d v_{M}
$$

where $\operatorname{div}(V)$ denotes the divergence of $V$ and Ric is the Ricci tensor on $M^{m}$.

Now we apply the following formula of Yano $[6, \mathrm{p} .41,(1.11)]$.

$$
\int_{M}\left\{(\operatorname{div}(V))^{2}+\|\nabla V\|^{2}-\operatorname{Ric}(V, V)-\frac{1}{2}\left\|L_{V}(g)\right\|^{2}\right\} d v_{M}=0
$$

to obtain

$$
E_{V}^{\prime \prime}(0)=e^{m / 2} \cdot \int_{M} \frac{1}{2}\left\|L_{V}(g)\right\|^{2} d v_{M} \geqslant 0 .
$$

Clearly $E_{V}^{\prime \prime}(0)=0$ if and only if $L_{V}(g)=0$, that is, if $V$ is a Killing vector field. $\square$

\section{EXAMPLES OF UNSTABLE EXPONENTIALLY HARMONIC MAPS.}

First let us consider $f: M^{n-1} \rightarrow N^{n}$ an isometric and totally geodesic immersion of a hypersurface $M^{n-1}$ into $N^{n}$.

Let $u_{i}=f_{*} e_{i}$ for $i=1, \cdots, n-1$. Then $\left\{u_{1}, \cdots u_{n-1}\right\}$ forms an orthonormal basis of $T M$. Let $V$ denote a unit normal vector field of $M^{n-1}$ in $N^{n}$. Then $\left\{u_{1}, \cdots u_{n-1}, V\right\}$ forms an orthonormal basis of $T N$.

LEMMA 14. $\nabla_{u_{i}} V=0$ for $i=1, \cdots, n-1$.

PROOF: First from $\langle V, V\rangle=1$ we have $\left\langle\nabla_{u_{i}} V, V\right\rangle=0$.

Next from $\left\langle V, u_{j}\right\rangle=0$ for $j=1, \cdots, n-1$ we have $\left\langle\nabla_{u_{i}} V, u_{j}\right\rangle=-\left\langle V, \nabla_{u_{i}} u_{j}\right\rangle=$ $-\left\langle V, B_{M}\left(u_{i}, u_{j}\right)\right\rangle=0$ where $B_{M}$ denotes the second fundamental form of $M^{n-1}$ in $N^{n}$, which is zero in this case since $M^{n-1}$ is totally geodesic. 
Applying Lemma 14 to Proposition 10, we obtain

$$
E_{V}^{\prime \prime}(0)=e^{(n-1) / 2} \int_{M}-\operatorname{Ric}_{N}(V, V) d v_{M}
$$

where $\operatorname{Ric}_{N}$ is the Ricci tensor on $N^{n}$.

This proves the following.

THEOREM 15. Let $f: M^{n-1} \rightarrow N^{n}$ be an isometric and totally geodesic immersion of a hypersurface $M^{n-1}$ into $N^{n}$. If the Ricci curvature of $N^{n}$ is positive then $f$ is an unstable exponentially harmonic map.

A similar calculation as above proves the following

TheOREM 16. Let $f: M^{m} \rightarrow N^{n}, m<n$ be an isometry. Assume that $N^{n}$ has positive sectional curvatures. If there exists a vector field $V$ normal to $f(M)$ and parallel along $f(M)$, then $E_{V}^{\prime \prime}(0)<0$ and hence $f$ is an unstable exponentially harmonic map.

Theorem 2 now follows from Theorem 13 and Theorem 16.

\section{REFERENCES}

[1] M.C. Hong, 'On the conformal equivalence of harmonic maps and exponentially harmonic maps', Bull. London Math. Soc. 24 (1992), 488-492.

[2] H.B. Lawson, Minimal varieties in real and complex geometry, Séminaire de Mathématiques Supérleures 57 (Les Presses de l'universite de Montreal, Montreal, Que., 1974).

[3] P.F. Leung, 'A note on stable harmonic maps', J. London Math. Soc. (2) 29 (1984), 380-384.

[4] R.T. Smith, 'The second variation formula for harmonic mappings', Proc. Amer. Math. Soc. 47 (1975), 229-236.

[5] H. Takeuchi, 'Stability and Liouville theorems of p-harmonic maps', Japan J. Math. 17 (1991), 317-332.

[6] K. Yano, Integral formulas in Riemannian geometry, Pure and Applied Mathematics 1 (Marcel Dekker, New York, 1970).

Department of Applied Mathematics

Hong Kong Polytechnic University

Hung Hom

Kowloon

Hong Kong

e-mail: matheclf@maun01.ma.polyu.edu.hk
Department of Mathematics

National University of Singapore

Kent Ridge

Singapore 119260

e-mail: matfred!@leonis.nus.edu.sg 